\title{
First presentation of Crohn's disease complicated with hepatic portal venous gas and pylephlebitis
}

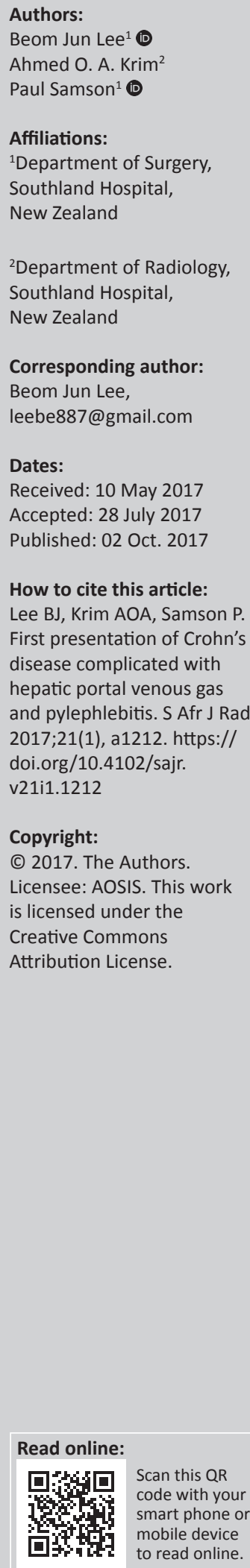

The authors present a rare case where radiological findings provided an important diagnostic and prognostic role in a surgical patient with abdominal sepsis. The case in interest describes an extremely rare surgical complication of intestinal Crohn's disease (CD), namely, pylephlebitis and hepatic portal venous gas (HPVG). Key radiological findings and their clinical significance are described to further add to the limited published data available on $\mathrm{CD}$ with pylephlebitis and HPVG.

\section{Introduction}

Pylephlebitis, a condition characterised by suppurative thrombosis of the portal vein or its branches, is an extremely rare and often fatal complication of infections in gastrointestinal structures drained by the portal venous system. ${ }^{1,2}$ Micro-perforation and ulceration of intestinal mucosa, or disease invasion deeper into the submucosal layer, can lead to the development of small vessel thrombophlebitis which can extend to the portal venous system. Furthermore, similar pathogenesis can lead to the escape of bowel gas into the portal system forming hepatic portal venous gas (HPVG), a rare radiological sign that is often associated with a severe clinical course. ${ }^{3}$ New-onset intestinal Crohn's disease (CD) with concurrent development of pylephlebitis with or without HPVG is extremely rare, with only seven cases previously described in the literature. ${ }^{4}$

\section{Case presentation}

A 39-year-old woman presented to the emergency department with acute right-sided abdominal pain in association with fever. She had no previous surgical or medical history and her family history was unremarkable. On examination, she was septic with a non-distended abdomen and right-sided abdominal tenderness. An abdominal ultrasound scan to investigate for cholecystitis showed gas bubbles in the portal vein branches (Figure 1). Contrast-enhanced abdominal computed tomography (CT) showed circumferential mural thickening and enhancement of the terminal ileum with prominent perienteric vasculature, resulting in a comb sign. The CT scan confirmed the presence of portal venous gas with diffuse portal venous wall thickening and multiple filling defects in distal branches consistent with pylephlebitis (Figure 2).

Despite initial conservative management with broad spectrum antibiotics, the patient deteriorated with clinical signs of peritonism, and blood tests reported markedly elevated white cell count plus inflammatory markers. The patient, therefore, proceeded to emergency laparotomy. Intra-operatively a grossly thickened terminal ileum with mesenteric wrapping was found (Figure 3). An ileocolic resection with stapled side-to-side primary anastomosis was performed. Pathological examination showed transmural inflammation of the terminal ileum consistent with CD (Figure 4). Follow-up CT scan of the abdomen performed on day seven post-operatively showed complete resolution of the HPVG and pylephlebitis. The patient was discharged with no surgical complications.

\section{Discussion}

Both pylephlebitis and HPVG are rare complications of intra-abdominal inflammation. Inflammatory bowel disease (IBD), such as $\mathrm{CD}$, has been reported to be a rare but important aetiology of these complications. ${ }^{4}$ The difficulty in diagnosing IBD can be compounded by such complications, specifically pylephlebitis, as this may present with vague symptoms of visceral abdominal pain, chronic fever and, in advanced cases, jaundice and hepatosplenomegaly. ${ }^{2}$ Both pylephlebitis and HPVG are considered life-threatening radiological signs that correlate with the severity of the underlying abdominal disease and ultimately are associated with a high mortality rate (up to $39 \%$ ). ${ }^{2}$ 
Delay in treatment of pylephlebitis can lead to hepatic abscess, mesenteric infarction and bowel necrosis.

Pylephlebitis diagnosis relies on the demonstration of thrombus in the portal mesenteric system in the setting of

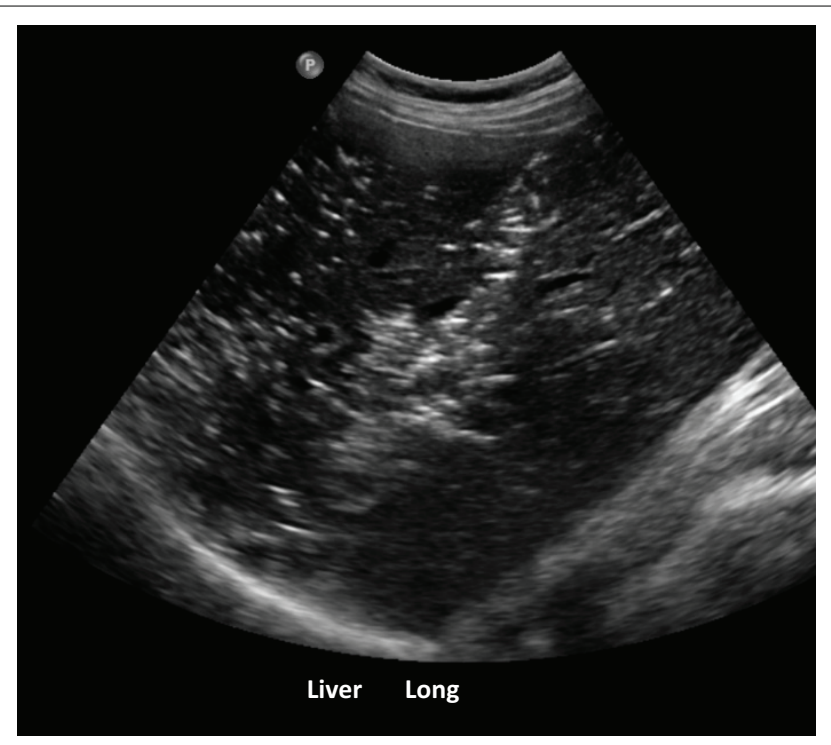

FIGURE 1: Ultrasound showing portal venous gas as diffuse echogenic foci and reflector shadows in the liver.

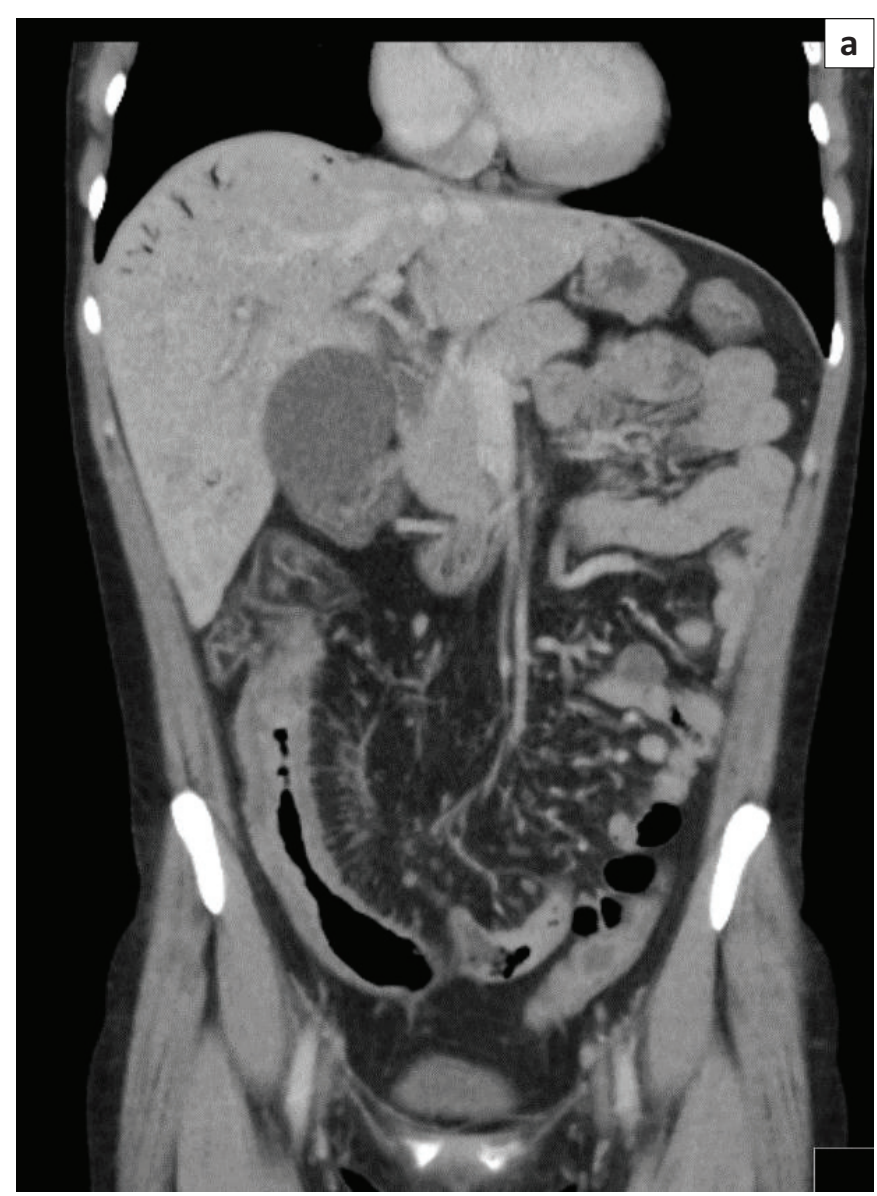

suppurative bacteraemia. As clinical presentation is relatively non-specific, radiological studies play an essential role in diagnosis. Portal venous phase CT scan is the diagnostic modality of choice. Imaging findings are influenced by the site of portal venous involvement, the primary source of infection and intra-abdominal complications. Liver imaging findings include unopacified branches of the portal vein,

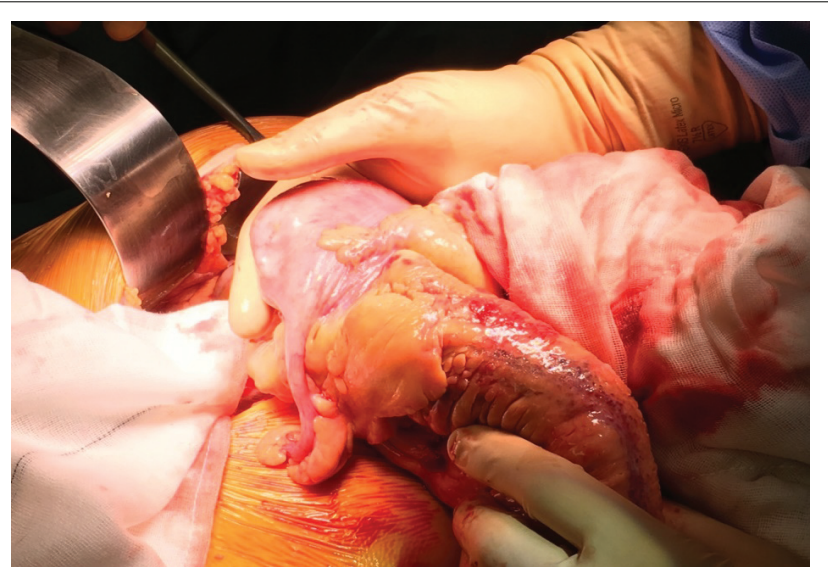

FIGURE 3: Intra-operative view demonstrating a grossly thickened, oedematous terminal ileum with serosal inflammation and omental wrapping.

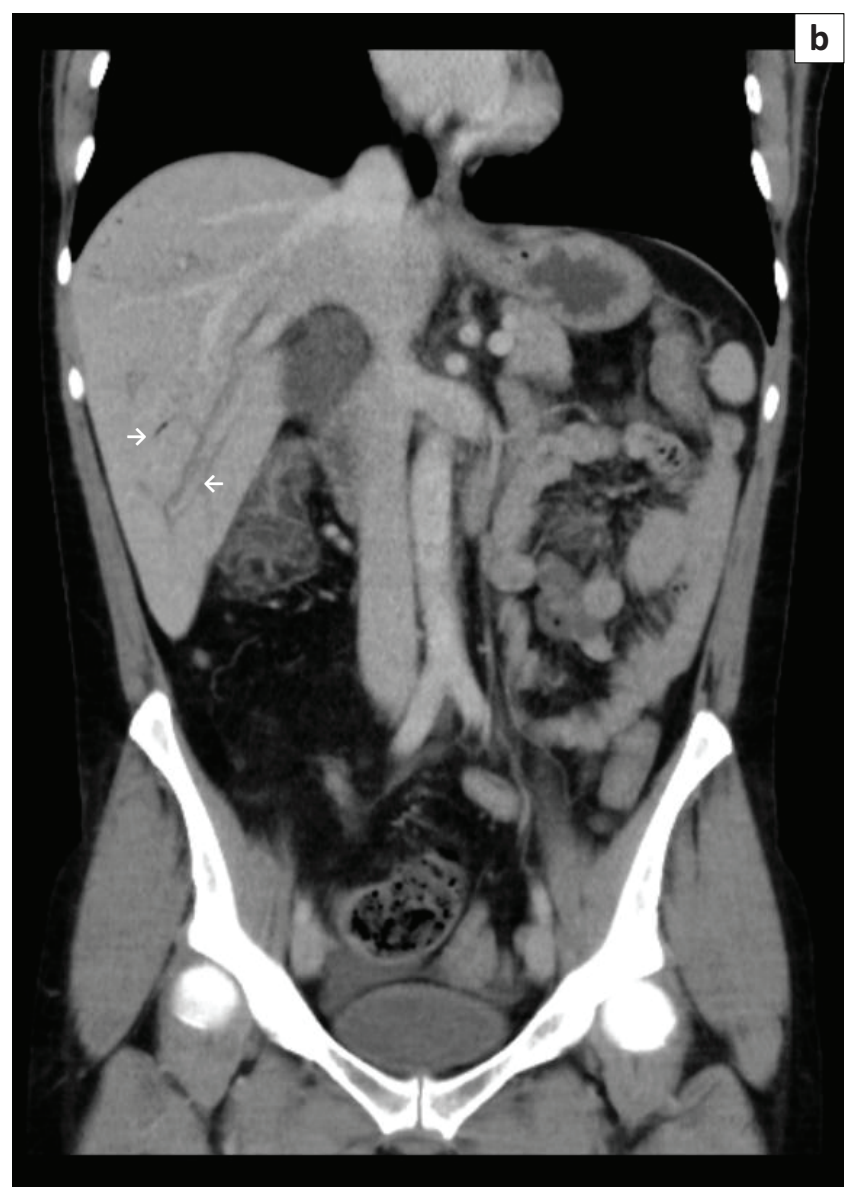

FIGURE 2: Coronal images from a CT abdomen demonstrating (a) circumferential mural thickening and enhancement of the terminal ileum with prominent vasculature resulting in a comb sign (b) portal venous gas with associated portal venous wall thickening and multiple filling defects in distal portal vein branches (arrows) consistent with pylephlebitis. 


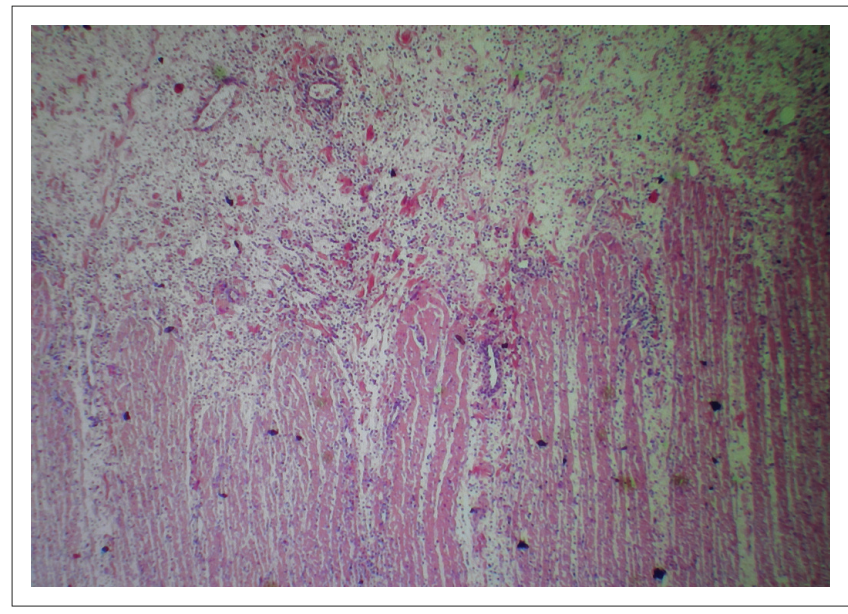

FIGURE 4: Histology of the terminal ileum, demonstrating inflammatory cell invasion through all layers of the intestine and crypt distortion, which is consistent with Crohn's disease (Hematoxylin-eosin x 10).

transient hepatic attenuation differences (THAD) and intrahepatic abscesses. ${ }^{5}$

Hepatic portal venous gas is readily characterised by linear or dendritic radiolucencies along the periphery of the liver on CT scan or echogenic foci on ultrasound. This is in contrast to air in the biliary tree (pneumobilia) that has a central distribution and remains an important differential diagnosis. While HPVG is a critical radiological sign with associated high mortality, recent reports have suggested that HPVG in patients with $\mathrm{CD}$ does not necessarily indicate necrotic bowel. ${ }^{6}$ Our case supports this statement. In addition, we have demonstrated that after the successful surgical treatment of CD, both HPVG and pylephlebitis can be resolved as early as within a week. The role of anticoagulation in the treatment of pylephlebitis remains controversial. ${ }^{24}$ In our case a successful clinical and radiological resolution of pylephlebitis was achieved shortly after the surgical management of the primary bowel disease and antibiotic therapy without the use of anticoagulation.

Crohn's disease is often initially managed medically unless there is fulminant sepsis, mechanical complications such as bowel obstruction or perforation. Our case demonstrates that the presence of either pylephlebitis or HPVG should warrant early surgical consultation and consideration of operative intervention. We thus propose that in patients with severe operable CD, complicated by HPVG and pylephlebitis, early resection of the affected intestinal segment is a valid, definitive treatment option.

\section{Conclusion}

Radiological investigations play an essential role in the diagnosis of first presentation of $C D$ with its rare complications of HPVG and pylephlebitis. Early surgical intervention may be necessary in some patients for better outcomes to be achieved.

\section{Acknowledgements}

This research was performed in the Department of Surgery and Department of Radiology, Southland Hospital, New Zealand. The patient's consent and confidentiality was respected at all times.

\section{Competing interests}

The authors declare that they have no financial or personal relationship(s) that may have inappropriately influenced them in writing this article.

\section{Authors' contributions}

B.J.L. (Surgica Registrar) was the project leader who wrote the majority of the article. A.O.A.K. (Consultant Radiologist) was responsible for providing radiological figures and legends. P.S. (Consultant General Surgeon) was responsible for overseeing the manuscript and providing surgical perspective of the case.

\section{References}

1. Wong K, Weisman DS, Patrice KA. Pylephlebitis: A rare complication of an intraabdominal infection. J Community Hosp Intern Med Perspect. 2013;3(2). https:// doi.org/10.3402/jchimp.v3i2.20732

2. Choudhry AJ, Baghdadi YM, Amr MA, Alzghari MJ, Jenkins DH, Zielinski MD. Pylephlebitis: A review of 95 cases. J Gastrointest Surg. 2016;20(3):656-661. https://doi.org/10.1007/s11605-015-2875-3

3. Liebman PR, Patten MT, Manny J, Benfield JR, Hechtman HB. Hepatic - Portal venous gas in adults: Etiology, pathophysiology and clinical significance. Ann Surg. 1978;187(3):281-287. https://doi.org/10.1097/00000658-197803000-00012

4. Shin AR, Lee CK, Kim HJ, et al. Septic pylephlebitis as a rare complication of Crohn's disease. Korean J Gastroenterol. 2013;61(4):219-224. https://doi.org/10.4166/ kjg.2013.61.4.219

5. Balthazar EJ, Gollapudi P. Septic thrombophlebitis of the mesenteric and portal veins: CT imaging. J Comput Assist Tomogr. 2000;24:755. https://doi.org/ 10.1097/00004728-200009000-00017

6. Abboud B, El Hachem J, Yazbeck T, Doumit C. Hepatic portal venous gas: Physiopathology, etiology, prognosis and treatment. World J Gastroenterol. 2009;15(29):3585-3590. https://doi.org/10.3748/wjg.15.3585 